\title{
Imaging findings of an asymptomatic child with pulmonary vein atresia: reply to Gasparetto
}

\author{
Mindaugas Mataciunas
}

Received: 8 March 2010 /Accepted: 18 March 2010/Published online: 1 May 2010

(C) Springer-Verlag 2010

Sir,

Gasparetto et al. [1] present an interesting case report of a rare anomaly - asymptomatic unilateral pulmonary vein atresia that was confirmed by magnetic resonance angiography. Unlike the case reported at our institution [2], the girl had no symptoms and on follow-up after 5 years she remained asymptomatic. An asymptomatic or mildly symptomatic course of this anomaly could be explained by complexity and pliability of the bronchopulmonary circulation. Liebow [3] showed a double venous drainage in normal human lungs consisting of pulmonary and bronchial veins that intercommunicate between each other. Proximal bronchial veins drain into the azygos, hemiazygos and intercostal veins and pulmonary veins drain into the left atrium. Presumably connections between the pulmonary veins and the caval system via azygos veins are capable of expansion or proliferation in various congenital or acquired pathological conditions. Definitive diagnosis and functional parameters of this anomaly require invasive angiography, but because the girl is asymptomatic, postponement of this procedure could be justifiable. We suggest that increasing availability of cross-sectional techniques (CT, MR) with comprehensive three-dimensional visualization will be used more frequently as a first-line modality to clarify incidental findings. Therefore it is possible that this cardiovascular defect will appear to be not so infrequent.

\section{References}

1. Gasparetto TD, Daltro P, Marchiori E (2010) The imaging findings of an asymptomatic child with pulmonary vein atresia. Pediatr Radiol 40. doi:10.1007/s00247-010-1663-1

2. Mataciunas M, Gumbiene L, Cibiras S et al (2009) CT angiography of mildly symptomatic, isolated, unilateral right pulmonary vein atresia. Pediatr Radiol 39:1087-1090

3. Liebow AA (1953) The bronchopulmonary venous collateral circulation with special reference to emphysema. Am J Pathol 29:251-289
M. Mataciunas $(\square)$

Clinic of Thoracic Diseases, Allergology and Radiology,

Faculty of Medicine, Vilnius University,

M.K. Ciurlionio 21,

Vilnius 03101, Lithuania

e-mail: mindaugas.mataciunas@santa.lt 